\title{
Design \& Inspection of Voltage Stabilization Circuit using 3tscmi
}

\author{
G Gnaneshwar Kumar, K Karunanithi
}

\begin{abstract}
The electrical industries have more concern regarding the development of inverters and its improvisation, which is drastically growing day-by-day. The need of getting more and more power from inverters is the major requirement and attaining that is a major task for every researchers/developers/engineers. This paper, investigates and experimentally proves the purpose and needs of Multi-Level Inverter circuit design, which is one of the best solution to provide heavy-yield as well as less-noise level compare to other inverters. In this paper, a new strategic design called 3-Phase Transformer Supported-Cascaded-Multi-Level Inverter (3TSCMI), which is introduced to attain many useful benefits. That kind of benefits is listed in detail: (i) Power-Emission with Noise-Free strategies, (ii) achieves heavy-power-yielding, (iii) Maintains low-Frequency level and many more. The purpose of 3TSCMI is to be explored by means of strategic principles, those are useful to improve the efficiency of circuit designing, such as: (i) Extracting the requirement of Cascaded Multi-Level Inverter, (ii) Identify the best power-source option of the circuit, (iii) implementing cross-ideas to establish the uni-source $D C$ power acquirement from PhotoVoltaic, (iv) Identify the pastimplementation strategies and devise a plan to reemployment that in new one with resolved issues and (v) Designing the 3TSCMI circuit with Active Power Filters. The Active Power Filters are used to improve the efficiency of 3TSCMI by means of reducing the noise level and produce the proper power-yield with accurate manner. Along with this an advanced Voltage Source Converters are used into the designing of 3TSCMI circuit nature, for establishing the performance better and attaining the highefficient power yielding. The source voltage accumulation is done by using windmill power source, which reduces the cost wastages and improve the benefit structure of new modeled circuit as well as makes the entire design to be perfect and producing best yield compare to other classical approaches.
\end{abstract}

Keywords: Active Power Filter, Cascaded Multi-Level Inverter, CMI, 3-Phase Transformer Supported-CMI, 3TSCMI, Voltage Source Converter.

\section{INTRODUCTION}

Now-a-days, the importance of power-electronics-anddrives in power-distribution-environment achieves a vast improvement and demands globally. The general purpose power-electronic-devices are normally considered to used in converters/inverters to transform the energy into required energy form, which is required for power-grids with respect to voltage-and-frequency. The renewable power sources such as Photovoltaic, Windmill and others are commonly used to act as a supplement power producing mediums to

Revised Manuscript Received on July 10, 2019.

G GNANESHWAR KUMAR, Research Scholar, School Of Electrical \& Communications, Vel Tech Rangarajan Dr.Sagunthala R\&D Institute of Science and Technology, Avadi, Chennai 600 062, T.N, India.

K KARUNANITHI, Associate Professor, School Of Electrical \& Communications, Vel Tech Rangarajan Dr.Sagunthala R\&D Institute of Science and Technology, Avadi, Chennai 600 062, T.N, India resolve the cost problems and other power wastages, which are commonly associated with converters and it is utilized to associate the generator-to-grid.

The most-known Maximum power Point Tracking (MPPT) principle is applied to convert the output power of Solar-Photovoltaic into required form of DC power. Then Outputted DC power will be changed top required form of $\mathrm{AC}$ power and it is used for commercial and noncommercial usages. The Multi-Level Inverters are considered to be a boom to the electrical industry, which produces great and heavy-power-yield with less-noise strategies. All the commercial and non-commercial purpose and business and normal individuals are required to implement the purpose of Multi-Level Inverters for their usages. The necessity of Multi-Level-Inverter circuit planning is increasingly and additionally heaps of analysts work consistently in this field to achieve best yields from those multi-level-inverters.

The conventional methodologies trust more in fell multilevel-inverter circuit outline and it delivers sufficient outcomes also in nature. The fundamental target of this paper is to experimentally demonstrate the proficiency of multi-level-inverters and imagine another measured circuit approach in view of CMI to give commotion free and ground-breaking yields. This paper presents another circuit plan nature in light of the customary fell multi-levelinverter, called 3-Phase Transformer Supported-CascadedMulti-Level Inverter (3TSCMI). This new plan is incorporated with the separating/filtering and converter circuits to accomplish more yields and precision in come about. With the relationship of Active Power Filters and Voltage Source Converters, the circuit can furnish best yields with less-clamor and high power yield. By executing this sort of shrewd circuits we can achieve more advantages, for example, enormous power-yields, disposing of the noise, diminishing the Electro-Magnetic Interference (EMI) and superior with less-clamor. 


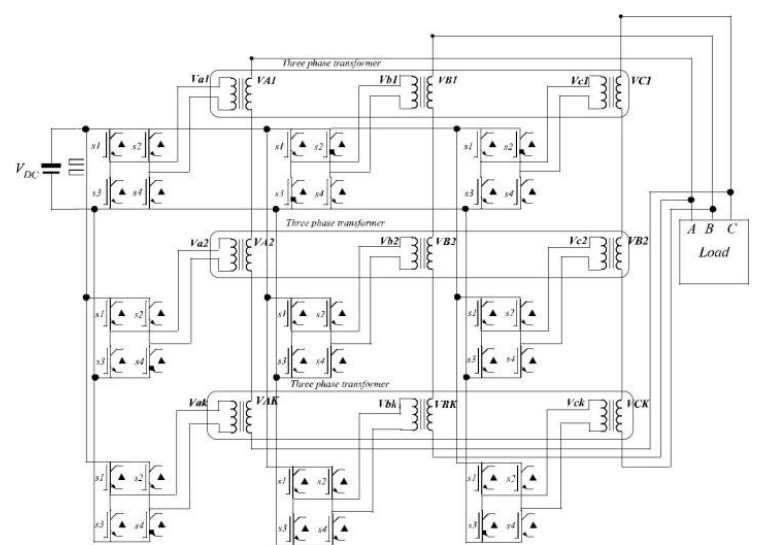

Fig.1. Circuit Model of Conventional CMI

The power-source is again thought about finished this approach, in customary methodologies bunches of energy sources are considered into account, however all are battled with specific cases, such sort of energy sources are batteries, fuel-products and some more. This situation drives the circuit to move with inexhaustible power sources, which are the most intense and appealing medium to outline the circuit in cost-productive and ground-breaking way. Other than in the transmission lines, high-control electronic frameworks are expected to guarantee the power dispersion and the vitality quality. Along these lines, control electronic inverters have the duty to do these undertakings with high proficiency.

\section{CONVENTIONAL MULTI-LEVEL INVERTER CIRCUIT PLAN-A SUMMARY}

In electrical industry there is dependably a colossal rivalry raises between-the utilization and necessities of customary converter topologies, for example, (i) HeavyVoltage customary Current Converter topology (HV) SemiConductors and (b) Medium Voltage (MV) modularized Converter Topology gadgets/contraptions. The accompanying figure shows the situation all the more plainly, for example, gadgets are serially master-minded with inverter and also which exhibits the customary converter and output waveform [4][5]. The Traditional Inverter Circuit configuration consists a few focal points, some of them are recorded beneath: (i) the conventional inverter circuit utilizes standard Pulse width Modulation (SPWM) procedure, (ii) Usage and amount of intensity circuit-parts are less contrast and other distinctive level circuit designs, (iii) Includes the repetitive levels by applying more serial gadgets than the real-needs, with the goal that the circuit can work even one getting fizzled and (iv) enhancing the circuit-unwavering quality. There are a few bad marks related with the conventional inverters, some of them are recorded underneath: (i) the entire-DC-voltage appears on each-switch, even it if in off-position, that will higher than the voltage of every gadget/contraptions, (ii) current-spillage, (iii) the gadgets won't share the voltage amid changing because of varieties in exchanging-speed, (iv) Numerous entryways/drives are required [7], (v) two level outcomes produce tremendous voltage steps, which makes an issue in motor/engine protection and (vi) circuitharmonics are huge.

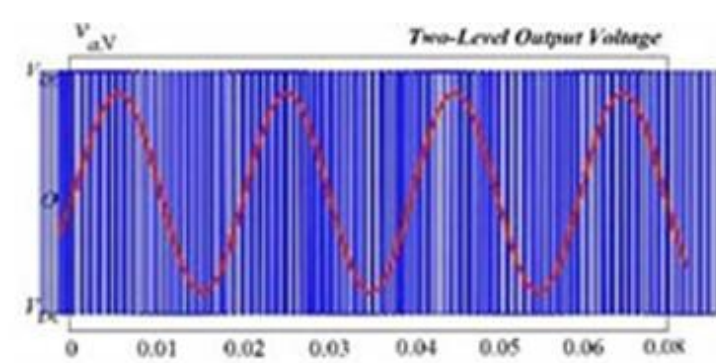

\section{Fig.2 Waveform of Conventional Multi-Level Inverter with 2-Loop Output Voltage}

\section{LITERATURE SURVEY}

In the year of 2017 the authors "Neha Sahu and Narendra D. Londhe [1]" proposed a paper titled "Selective harmonic elimination in five level inverter using sine cosine algorithm [1]" in that they concentrate more on designing a Multi level Inverter based Shunt-Type Active-Filtering, the designing is based on PhotoVoltaic (PV) based source for attaining medium voltage and high power distribution environment with changed controlling of inverter circuits under regular and disorted constraints. This circuit minimizes the reactivecurrent-stress and improves the power-distribution-quality with best timing. In this circuit designing faster dynamic response and enhanced phase-locked loop (EPLL) is applied for attaining good yields. However, the circuit complexity is increased because of the $\mathrm{H}$-Bridge circuit necessities and converter designing (ex. buck and boost converters), which also increases the cost and size of the circuit [10][11].

In the year of 2017 the authors "Mohammad Khenar, Amir Taghvaie, Jafar Adabi and Mohammad Rezanejad [2]" proposed a paper titled "Multi-level inverter with combined T-type and cross-connected modules [2]" in that they concentrated to design a Hybrid 9-Level Symmetric Cascaded Multilevel Converter with fault tolerant abilities. This paper proves that the proposed circuit is able to avoid fault scenarios and which it improves the reliability and availability of converter design during fault conditions. However, the circuit design needs to be improved based on the short-circuit fault detection scenarios, the researchers tried that in the paper but the experimental results not conclude this drawback was eliminated [6][8].

In the year of 2017 the authors "Khushaboo Rani Shandilya and Uma Shankar Patel [3]" proposed a paper titled "Mitigation of total harmonic distortion using cascaded MLI-DSTATCOM in distribution network [3]" in that they utilize the main advantage of Level-shifted-PulseWidth-Modulation scheme over Multi-Level-Inverter circuit design with Phase-Shifted logic, which drastically produces better performance compare to the existing works [11][12][13][14]. The core benefits attained from the proposed approach in this paper are reduced-harmonicdistortion, power-optimization, reducing the power-losses over high power and medium voltage electrical-applications The problem with this circuit designing is cost and time, because the designing of MLI circuit with LS-PWM and PS- 
PWM causes large devices to test the proposed circuit and practical implementations are complex in nature [12][15].

\section{TSCMI CIRCUIT DESIGNING - A SUMMARY}

The proposed approach obviously devises the arrangement to make a circuit, which is operable under great and creating best yield with commotion free imperatives. The proposed approach 3-Phase Transformer SupportedCascaded-Multi-Level Inverter (3TSCMI) can bolster longrun yield voltage abundance corrections and produces the best yield and current proportions with precise proportions. The proposed circuit configuration produces inconvenience free adjustment and requires less voltage control stockpiling medium. The lessening in voltage level in a roundabout way implies the diminishment in parts and current components, which likewise diminishing the normal mode-unsettling influences.

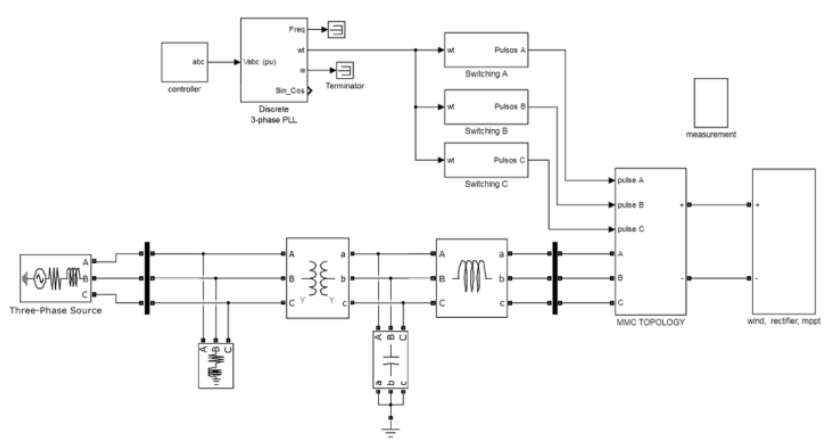

Fig.3 Proposed Circuit Design

Coming to structure point of view, each primary terminal of the transformer is connected to an H-Bridge module so as to synthesize output voltages of +VDC, Zero, -VDC. Every secondary of transformer is connected in series to enhance the output voltage level. Further, each phase terminal is delta connected to restrain the third harmonic component. The Circuit Design expresses that, primary of each phase is three phase and secondary is single phase terminal. All three terminals are series connected to generate phase voltage. Therefore, each phase can be expressed independently. As a result each phase multilevel inverter can be depicted as an isolated $\mathrm{H}$-Bridge cascaded multilevel inverter.

The proposed circuit outline of Cascaded-Multi-LevelInverter astutely uses the Unisource DC control supply from Windmill control source and works fine to create Medium voltage and High voltage control supply. A savvy ActivePower-Filter is intended to wipe out the commotion level brought up in 3-Phase Transformer Supported-CascadedMulti-Level Inverter and in addition Voltage-SourceConverter is intended to make the circuit more impeccable and to get more precise yields from the composed circuit.

\section{RESULT ANALYSIS}

The following table illustrates the input parameters of the proposed system circuit and mentioned the ranges of circuit.

Table.1 Input Parameters

\begin{tabular}{|c|c|}
\hline Parameter & Input-Level \\
\hline $\mathrm{L}_{\text {Line }}$ & $15 \mathrm{mH}$ \\
\hline $\mathrm{R}_{\text {Line }}$ & $10 \mathrm{Ohm}$ \\
\hline
\end{tabular}

\begin{tabular}{|c|c|}
\hline Lilter $_{\text {Initial Voltage of }}$ & $5 \mathrm{mH}$ \\
\hline $\begin{array}{c}\text { Transformer } \\
\text { Secondary-Voltage of } \\
\text { Transformer }\end{array}$ & $12000 \mathrm{Volt}$ \\
\hline $\begin{array}{c}\text { Frequency of Switching } \\
\text { units }\end{array}$ & $600 \mathrm{Volt}$ \\
\hline Active Load Power & $2 \mathrm{kHz}$ \\
\hline Reactive Load Power & $50 \mathrm{~kW}$ \\
\hline PF-Range & $35 \mathrm{kVar}$ \\
\hline DC-Link-Voltage & 0.90 \\
\hline
\end{tabular}

The design plan of 11 Level Multi-Level inverter designed in MATLAB/Simulink. Reproduction was 20s \& which contains serious inclining of breeze turbine. By doing this the objective will be to survey the conduct of the control framework in most exceedingly terrible. Table-1 demonstrates estimations utilized parameters for recreation. On or before time, $\mathrm{t}=6 \mathrm{~s}$, no breeze to control the breeze turbine; in this manner, the dc supply connect is open circuited. At time, $\mathrm{t}=6 \mathrm{~s}$, intensity of the inverter is increased by $12 \mathrm{~kW}$ out of $5 \mathrm{~s}$, and afterward sloped down to $3.5 \mathrm{~kW} 4 \mathrm{~s}$ later. The circuit diagram demonstrates the yield dynamic capacity from the breeze turbine. In the reenactment, the nearby load changes the Power factor PF to 0.82 . At this point when reproduction begins, the MLI make the Power Factor PF to 0.90. Figure-4 demonstrates the yield dynamic and receptive power from the breeze turbine and the lattice.

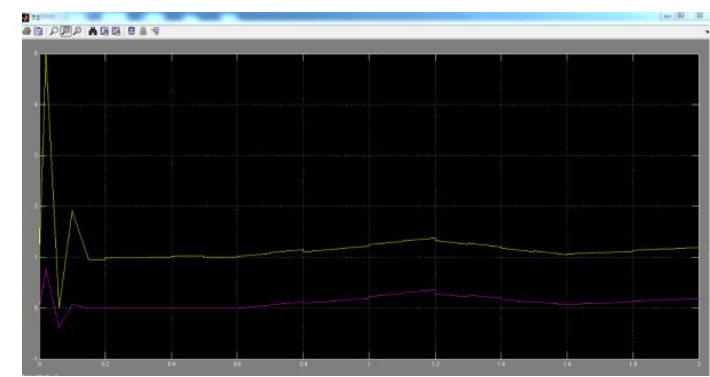

(a)

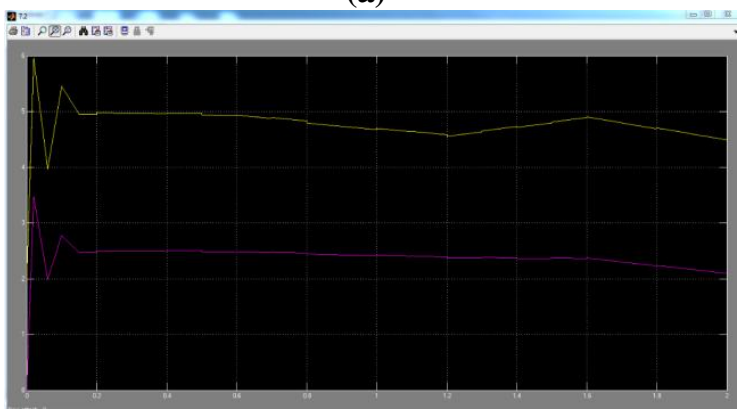

(b)

Fig.4 Simulation Waveform of (a) Inverter's $P$ and $Q$, (b) Grid's $\mathbf{P}$ and $\mathbf{Q}$

After $t=6 s$, the yield intensity of the breeze turbine is expanded, and thus the dynamic power gave by the line is decreased to a similar sum. The reenacted yield power of the inverter before the channel appeared in Figure-5. 


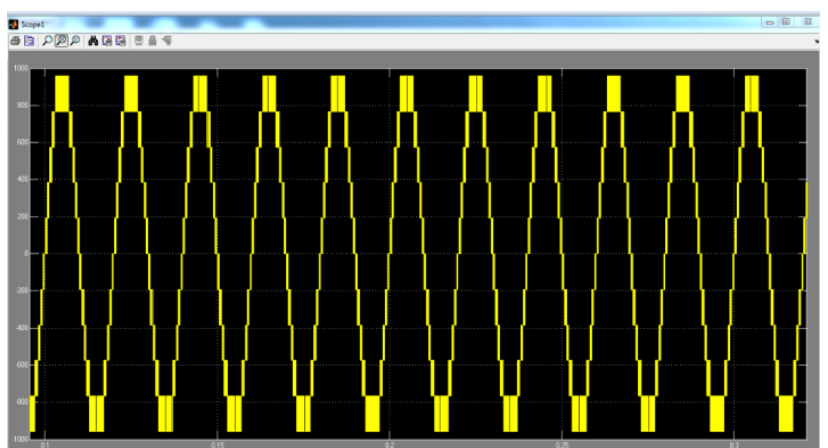

Fig.5 output - waveform for voltage of 11 Level MLI

The following figure, Figure- 6 demonstrates the PF of the matrix. The PF of the matrix is steady at 0.90 paying little heed to the dynamic power from breeze turbine, by which the fundamental objective of the inverter circuit is demonstrated. The set point for dc interface of the inverter is $2000 \mathrm{~V} \&$ the RMS estimation of yield potential is $600 \mathrm{~V}$.

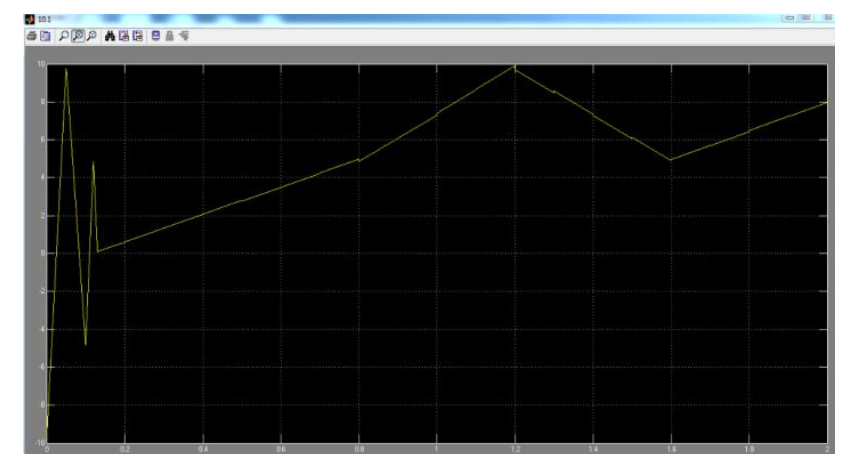

Fig.5 Simulated-Waveform of Delta \& Modulation index of 11 Level-Inverter

Figure- 6 demonstrates the PF of the lattice. The PF of the lattice is consistent at 0.90 paying little respect to the dynamic power capacity from the breeze turbine. When the dynamic power originates from breeze turbine machine, the control framework expands the estimation of the yield edge so as to get more dynamic capacity to the matrix. In this way, the dynamic power gave from the feeder lines to the heap are diminished, $\&$ therefore receptive yield from the feeder is diminished. Thusly, the tweak record is expanded by the control framework to infuse maximum responsive yield required by the heap. By this, a scaled adaptation of the proposed MLI has been manufactured and tried. The rating of Power of this model is $250 \mathrm{~W}$ or potentially VAR. Keeping this in mind that end goal which is to execute the control technique and to deal with the input signals, two CLP 1104 dSPACE frameworks which have been synchronized. A 3 stage Permanent Magnet DC generator driven by a variable speed dc engine is utilized to imitate the breeze turbine changes speed. The following figure demonstrates the framework contents before remuneration in which the MLI is detached from lattice. Then the MLI is open circuited and no dynamic or responsive power exchange $b / w$ the breeze emulator and the inverter.

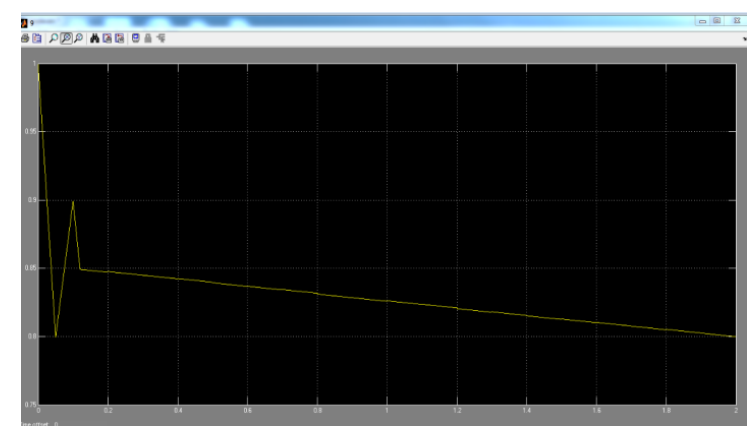

Figure.6 Simulated-Waveform of PF-Grid

\section{CONCLUSION AND FUTURE WORK}

The proposed approach produces a good enough circuit design to complete the working of Cascaded-Multi-LevelInverter with modified nature. The proposed circuit design follows the new approach called "3-Phase Transformer Supported-Cascaded-Multi-Level Inverter (3TSCMI)", which can provide complete support to outcome long-range output voltage and frequency amendments as well as generates accurate power ratios. This proposed system clearly demonstrates and experimentally proves that the performance and efficiency of proposed circuit design called "3-Phase Transformer Supported-Cascaded-Multi-Level Inverter (3TSCMI)", which is beneficiary to attain heavyyield based on renewable power source and produces noisefree outcomes in efficient manner. The experimental consequences of the proposed circuit configuration ensure the inconvenience free adjustment and also which expends just less-voltage control stockpiling situations. The proposed circuit likewise works in view of less number of electrical and power parts, with the goal that the cost and size savvy recipient is additionally high contrast with the current methodologies. For all the proposed circuit configuration delivers best outcomes contrast with the current methodologies, which are unmistakably exhibited by means of similar outcomes in the prior cited synopsis. In future the circuit is more refined in light of Shunt-Active Power-Filter execution more than 3-Phase Transformer SupportedCascaded-Multi-Level Inverter alongside Polarized-PulseWidth-Modulation, which can enhance the general precision of the proposed configuration in light of clamor free figments and makes the circuit to work all the more splendidly.

\section{REFERENCES}

1. Neha Sahu and Narendra D. Londhe, "Selective harmonic elimination in five level inverter using sine cosine algorithm", IEEE International Conference on Power, Control, Signals and Instrumentation Engineering (ICPCSI), 2017

2. Mohammad Khenar, Amir Taghvaie, Jafar Adabi and Mohammad Rezanejad, "Multi-level inverter with combined T-type and cross-connected modules", IET Power Electronics, Volume: 11, Issue: 8-7-10, 2018.

3. Khushaboo Rani Shandilya and Uma Shankar Patel, "Mitigation of total harmonic distortion using cascaded MLIDSTATCOM in distribution network", IEEE International Conference on Power, Control, Signals and Instrumentation Engineering (ICPCSI), 2017.

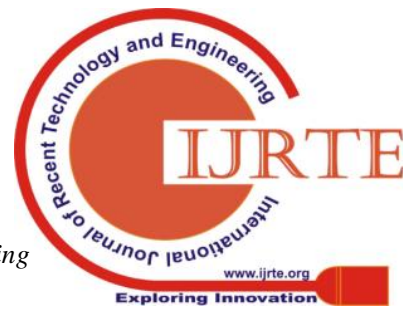


4. A. Beekmann, J. Marques, E. Quitmann, and S. Wachtel, "Wind energy converters with FACTS capabilities for optimized integration of wind power into transmission and distribution systems," in Proc. CIGRE/IEEE PES Joint Symp. Integr. Wide, Scale Renew. Resour. Power Del. Syst., Jul. 2009, pp. 1-9.

5. J. Rodriguez, J. S. Lai, and F. Z. Peng, "Multilevel inverters: Survey of topologies, controls, and applications," IEEE Trans. Ind. Appl., vol. 49, no. 4, pp. 724-738, Aug. 2002.

6. F. Z. Peng, J. S. Lai, J. W. McKeever, and J. VanCoevering, "A multilevel voltage-source inverter with separate DC sources for static VAr generation," IEEE Trans. Ind. Appl., vol. 32, no. 5, pp. 1130-1138, Oct. 1996.

7. L. M. Tolbert and F. Z. Peng, "Multilevel converters as a utility interface for renewable energy systems," in Proc. IEEE Power Eng. Soc. Summer Meeting, vol. 2. Jul. 2000, pp. 1271-1274.

8. S. Kouro, M. Malinowski, K. Gopakumar, J. Pou, L. G. Franquelo, B. Wu, et al., "Recent advances and industrial applications of multilevel converters," IEEE Trans. Ind. Electron., vol. 57, no. 8, pp. 2553-2580, Aug. 2010.

9. C. Tareila, P. Sotoodeh, and R. D. Miller, "Design and control of a single-phase D-STATCOM inverter for wind application," in Proc. PEMWA, Jul. 2012, pp. 1-5.

10. B. Gultekin and M. Ermis, "Cascaded multilevel converterbased transmission STATCOM: System design methodology and development of a $12 \mathrm{kV} \pm 12 \mathrm{MVAr}$ power stage," IEEE Trans. Power Electron., vol. 28, no. 11, pp. 4930-4950, Nov. 2013.

11. K. Sano and M. Takasaki, "A transformerless D-STATCOM based on a multivoltage cascade converter requiring no DC sources," IEEE Trans. Power Electron., vol. 27, no. 6, pp. 2783-2795, Jun. 2012.

12. X. Liang, Y. Xu, X. Chen, and C. Guo, "The simulation research of STATCOM based on cascaded multi-level converter," in Proc. 4th Int. Conf. Electr. Util. DRPT, Jul. 2011, pp. 494-498.

13. M. Davies, M. Dommaschk, J. Dorn, J. Lang, D. Retzmann, and D. Soerangr, HVDC PLUS Basic and Principle of Operation. Erlandgen, Germany: Siemens AG Energy Sector, 2009.

14. B. Gemmell, J. Dorn, D. Retzmann, and D. Soerangr, "Prospects of multilevel VSC technologies for power transmission," in Proc. IEEE Transmiss. Distrib. Conf. Exposit., Apr. 2008, pp. 1-16.

15. C. D. Barker and N. M. Kirby, "Reactive power loading of components within a modular multi-level HVDC VSC converter," in Proc. IEEE EPEC, Oct. 2011, pp. 86-90.

16. C. P. Tareila, "A single-phase D-STATCOM Inverter for distributed energy sources," M.S. thesis, Dept. Electr. Comput. Eng., Kansas State Univ., Manhattan, KS, USA, Aug. 2011.

17. Z. Yang, C. Shen, L. Zhang, M. L. Crow, and S. Atcitty, "Integration of a STATCOM and battery energy storage," IEEE Trans. Power Syst., vol. 16, no. 2, pp. 254-260, May 2001.

18. S. Chakraborty, B. Kroposki, and W. Kramer, "Evaluation of control and voltage regulation functionalities in a single-phase utility-connected inverter for distributed energy applications," in Proc. IEEE ECCE, Sep. 2009, pp. 1753-1759. 